\section{P531 CHEMSEX AND STI CLINIC USE AMONG MSM: RESULTS FROM A LARGE ONLINE SURVEY IN ENGLAND}

${ }^{1}$ Paula Blomquist*, ${ }^{2}$ Hamish Mohammed, ${ }^{2}$ Amy Mikhail, ${ }^{3}$ Peter Weatherburn, ${ }^{3}$ David Reid ${ }^{4}$ Sonali Wayal, ${ }^{2}$ Gwenda Hughes, ${ }^{4}$ Catherine Mercer. ${ }^{1}$ Public Health England, London, UK; ${ }^{2}$ Public Health England, National Infection Service, London, UK; ${ }^{3}$ London School of Hygiene and Tropical Medicine, Sigma Research, London, UK; ${ }^{4}$ University College London, Institute for Global Health, London, UK

\subsection{6/sextrans-2019-sti.609}

Background Chemsex, the use of select psychoactive drugs to enhance sexual experience, typically among men who have sex with men (MSM), is associated with STI risk behaviours. Understanding characteristics of MSM who engage in chemsex and their use of STI clinics is important for developing interventions.

Methods Between 5/2016-5/2017, 3,358 MSM (aged $\geq 15$ years; no known HIV infection) completed an online survey, largely via 4 gay social-networking apps. We described patterns of chemsex and differences in demographics, awareness of 8 common STIs, STI risk behaviour, and STI clinic attendance between those engaging in chemsex and those not. We used a composite measure 'high STI risk' comprising condomless anal sex (CAS) with $\geq 11 \mathrm{men} /$ past $3 \mathrm{~m}$, of whom $\geq 1$ had unknown HIV status. We used logistic regression to investigate the association between chemsex and clinic use/past $3 \mathrm{~m}$.

Results $8 \%$ of respondents reported chemsex/past year. Among them, $70 \%$ had used $\geq 2$ different chemsex drugs, with mephedrone $(68 \%)$ most popular. A greater proportion of MSM who reported chemsex, compared to those who did not, were university graduates (63\% vs $52 \%)$, <40years (51\% vs $43 \%$ ), aware of all 8 asked-about STIs (34\% vs 22\%), reported CAS/ past $3 \mathrm{~m}(73 \%$ vs $47 \%)$, and were classified as 'high STI risk' (26\% vs $8 \%$ ). MSM who reported chemsex were more likely to have attended an STI clinic/past $3 \mathrm{~m}$ vs those who did not (63\% vs 34\%), including after controlling for sociodemographics: adjusted odds ratio: 2.97, 95\%CI: 2.26-3.90. Of those at 'high STI risk' $(\mathrm{n}=317), 75 \%$ of MSM reporting chemsex, vs $48 \%$ of those not, had been to clinic/past $3 \mathrm{~m}$.

Conclusion A minority of MSM engage in chemsex. Those that do appear to be at greater STI risk but engage more actively with STI clinics. More targeted STI prevention efforts are needed to improve access to clinical services for all MSM at high risk of STIs.

Disclosure No significant relationships.

\section{P532 THE SEXUAL BEHAVIOUR AND HEALTH OF HETEROSEXUAL-IDENTIFYING MEN WHO HAVE SEX WITH MEN: A SYSTEMATIC REVIEW}

${ }^{1}$ Tyrone Curtis*, ${ }^{2}$ Kirsty Bennett, ${ }^{2}$ Lorraine Mcdonagh, ${ }^{1}$ Nigel Field, ${ }^{1}$ Catherine Mercer. ${ }^{1}$ University College London, Institute for Global Health, London, UK; ${ }^{2}$ University College London, Institute of Epidemiology and Health Care, London, UK

\subsection{6/sextrans-2019-sti.610}

Background Sexual behavioural and health differences are known to exist between gay and bisexual men, but less is known about heterosexual-identifying men who have sex with men (MSM). We conducted a systematic review of articles reporting on this population to inform public health interventions.

Methods We searched six databases for articles reporting sexual behaviour and health outcomes in heterosexual-identifying MSM in Western Europe, Australia, New Zealand and North
America, from 2008 to January 2018. All were screened by a primary reviewer, $10 \%$ were screened by a second independent reviewer.

Results From 3126 articles identified, 42 were quantitative and included in a narrative synthesis (40 reported on studies conducted in the USA). The majority reported data from MSM-focused studies; five were general population studies. HIV prevalence for heterosexual-identifying MSM (range across studies: 4.6-11.4\%) was lower than for gay (11.243.8\%) or bisexual (12.4-29.8\%) MSM, however, fewer heterosexual-identifying MSM reported recent testing for HIV (40$49 \%$ vs $64-68 \%$ of gay men, $56-62 \%$ of bisexual men) or STIs. There was no difference by sexual identity in MSM's reporting of recent condomless sex with casual male partners (42\%-52\% of those reporting recent sex with casual partners) or insertive condomless sex, however heterosexual-identifying MSM were less likely than gay MSM to report receptive condomless sex. They reported fewer recent male partners than gay or bisexual MSM, more recent female partners than gay MSM, and similar numbers of lifetime partners of either sex compared to gay or bisexual MSM.

Conclusion Heterosexual-identifying MSM report fewer male partners than gay or bisexual MSM, however similarities in risk behaviours indicate a group at risk of poorer sexual health than the general population. The data also suggest inadequate sexual health service use by these men such that additional targeted approaches to health promotion and infection control for this population may be warranted.

Disclosure No significant relationships.

\section{P533 HEPATITIS A VACCINE UPTAKE AMONG MEN WHO HAVE SEX WITH MEN FROM A TARGETED VACCINATION PROGRAM IN MELBOURNE IN 2018}

${ }^{1}$ Sam Burrell, ${ }^{2}$ Lenka Vodstrcil ${ }^{*},{ }^{2}$ Christopher Fairley, ${ }^{1}$ Alex Kilner, ${ }^{2}$ Catriona Bradshaw ${ }^{1}$ Marcus Chen, ${ }^{2}$ Eric Chow. 'Alfred Health, Melbourne Sexual Health Centre, Carlton, Australia; ${ }^{2}$ Monash University, Central Clinical School, Carlton, Australia

\subsection{6/sextrans-2019-sti.611}

Background In response to an outbreak of hepatitis A in men who have sex with men (MSM) in Victoria, Australia in 2017; the Victorian government funded a free hepatitis A vaccination program for all Victorian MSM in 2018. This study aimed to determine hepatitis A vaccine uptake among MSM in a sexual health clinic in Melbourne during the program, and assess the factors associated with vaccine uptake.

Methods All MSM attending the Melbourne Sexual Health Centre (MSHC) in 2018 were included in the analysis. Chart review was performed to determine why men did not receive the vaccine and the proportion of men vaccinated for hepatitis A was calculated. Multivariable logistic regression was then performed to examine the factors associated with vaccine uptake.

Results There were 9,582 MSM who attended MSHC in 2018, of whom 61.3\% $(\mathrm{n}=5,869, \quad 95 \% \mathrm{CI}: 60.3-62.2 \%)$ reported already being immune to hepatitis $\mathrm{A}$, either from previous vaccination or infection. Of the 3,713 remaining eligible men, $62.7 \%(n=2,327,95 \%$ CI:61.1-64.2\%) received the hepatitis A vaccine. Compared with HIV-negative MSM not taking PrEP, MSM taking PrEP $(\mathrm{aOR}=1.28$; 95\%CI:1.011.62) were more likely to receive the vaccine. In addition, vaccine uptake was associated with being aged 16-25 years $(\mathrm{aOR}=2.49 ; 95 \% \mathrm{CI}: 2.06-3.02)$ and $26-35$ years $(\mathrm{aOR}=2.10$; 
95\%CI:1.76-2.50) compared to being aged $\geq 35$ years. 1,386 men did not receive the vaccine. Of these men, the majority $(55.4 \% ; n=768)$ were not offered the vaccine by their treating clinician, and $21.6 \%(\mathrm{n}=300)$ were identified as nonimmune after serological testing but did not return for vaccination. By the end of 2018 , a total of $85.5 \%$ of MSM $(8,196 / 9,582)$ were immune to hepatitis A.

Conclusion The critical vaccination threshold for hepatitis A has been estimated at $\geq 70 \%$. Continuation of the targeted hepatitis A vaccination program will improve immunity amongst the MSM population to prevent ongoing transmission and the likelihood of future outbreaks.

Disclosure No significant relationships.

\section{P535 CHEMSEX \& AMP; SEXUAL CONSENT: A QUANTITATIVE STUDY ON SEXUALIZED DRUG USE IN MEN WHO HAVE SEX WITH MEN AND NON-CONSENSUAL SEX}

${ }^{1}$ Jilke Speulman, ${ }^{1}$ Susanne Druckler*, ${ }^{1}$ Martijn Van Rooijen* ${ }^{*}{ }^{2}$ Henry De Vries. ${ }^{1}$ Public Health Service of Amsterdam, Infectious Diseases, Amsterdam, Netherlands; ${ }^{2}$ Public Health Service Amsterdam, Amsterdam University Medical Center (UMC), National Institute of Public Health and the Environment (RIVM), Infectious Diseases, Infection and Immunity Institute (Al and II), Epidemiology and Surveillance Unit, Amsterdam, Netherlands

\subsection{6/sextrans-2019-sti.612}

Background Chemsex, the use of drugs (including crystal methamphetamine, mephedrone and/or GHB/GBL) to enhance sex, is practiced by men who have sex with men (MSM) and has risen in the last decade. Non-consensual sex is hypothesized to occur frequently under the influence of chemsex, however has not been investigated much. In this study we aimed to quantify chemsex use in the Amsterdam area and to establish whether non-consensual sex is associated with chemsex engagement.

Methods During the Amsterdam Pride in 2016 and 2018, Amsterdam-located users of a gay dating application were asked about chemsex behavior in the previous 6 months and non-consensual sexual experiences in the past 5 years (the latter in 2018). Non-consensual sex was defined as 'non-consensual sex or experience (e.g. filmed/photographed without consent)'. $\mathrm{X}^{2}$ test for independence was used for statistical analyses.

Results A total of 1833 (2016) and 756 (2018) participants were included of which $28.3 \%$ and $27.8 \%$ engaged in chemsex $(p=0.81)$. In 2018 the occurrence of non-consensual sex was high, but not significantly different among the chemsex $(20.4 \%)$ and non-chemsex $(16.5 \%)$ group $(p=0.22)$. Yet, among those with chemsex being filmed/photographed without consent, taking drugs against ones will and passing out, were reported more often than among those without chemsex $(\mathrm{p}<0.05)$. The intensity of emotional distress related to nonconsensual sex was not significantly different between the chemsex and non-chemsex group $(\mathrm{p}=0.63)$.

Conclusion The proportion of MSM in Amsterdam who engage in chemsex is high but stable over 2016-2018. The proportion of MSM experiencing non-consensual sex is also high, but not associated with chemsex engagement, nor is the intensity of emotional distress related to chemsex. Nonetheless, the type of non-consensual experience differs between the chemsex and the non-chemsex group. Sexual healthcare professionals need to address chemsex use and non-consensual sex during consultations involving MSM and refer men if deemed necessary.

Disclosure No significant relationships.

\section{P538 HIGH HIV INCIDENCE AMONG MEN WHO HAVE SEX WITH MEN IN EIGHT CHINESE CITIES: RESULTS FROM A COHORT STUDY}

${ }^{1}$ Wenting Huang, ${ }^{2}$ Yehua Wang, ${ }^{3}$ Haidong Lu, ${ }^{3}$ Joseph Tucker, ${ }^{3}$ Weiming Tang* ${ }^{1}$ University of North Carolina at Chapel Hill Project-China, Guangzhou, China; ${ }^{2}$ UNC ProjectChina, Guangzhou, China; ${ }^{3}$ UNC Gillings School of Global Public Health, Chapel Hill, USA

\subsection{6/sextrans-2019-sti.613}

Background Knowing the HIV incidence is essential for providing timely intervention among key populations. However, HIV incidence data from men who have sex with men (MSM) in China are limited. In this study, we aim to measure HIV incidence among MSM in eight cities and investigate correlates with incidence.

Methods This study is a secondary analysis of a steppedwedged randomized control trial focusing on promoting HIV testing among Chinese MSM. MSM from eight cities in Guangdong and Shandong Province were recruited and followed from July 2016 to August 2017. Sexual behaviors and HIV testing activity were measured at baseline and during follow-up survey every three months. Participants who reported to have tested HIV at least twice during different follow-up periods were included in this analysis. We defined the seroconversion as a negative HIV testing result followed by a positive result during any of the follow up. We used Cox regression to examine correlates with HIV seroconversion.

Results Of the 1381 participants recruited at baseline, 360 MSM had HIV tests at least twice. At baseline, 87.5\% (315/ $360)$ were never married and $32.8 \%$ (118/360) never disclosed their sexual orientation to anyone other than their sexual partners. During the 12-month follow-up period, $56.67 \%$ (204/360) had multiple male partners, $15.3 \%$ (55/360) had female partners and $14.7 \%$ (53/360) had bisexual behaviors. Overall, 25 men had seroconversion, and the HIV incidence rate was 15.0/100 person-year. The Cox regression model showed higher seroconversion risk was associated with a higher number of female sexual partners $(\mathrm{HR}=3.59$, 95\% $\mathrm{CI}$ : 1.32-9.80). The association between seroconversion and bisexual behavior was marginally significant $(\mathrm{HR}=2.05$, 95\% $\mathrm{CI}$ : 0.98-4.29).

Conclusion HIV incidence is very high among MSM in our study based in China. It was associated with multiple sexual partners, as well as bisexual behaviors. Interventions to prevent HIV transmission, targeting on sexual behavior change are needed.

Disclosure No significant relationships. 